\title{
EL APRENDIZAJE BASADO EN PROYECTOS (ABP) COMO METODOLOGÍA DE TRABAJO EN EL AULA UNIVERSITARIA DE LENGUA ESPAÑOLA CON ALUMNADO ITALIANO
}

\section{THE PROJECT BASED LEARNING (PBL) AS METHOLOGY OF WORK AS UNIVERSITY CLASSROOM OF SPANISH LANGUAGE WITH ITALIAN STUDENTS}

\section{Zósimo López Pena}

\section{Resumen}

El presente artículo tiene como objetivo la presentación de los resultados de la investigación y la experiencia didáctica llevada a cabo en la asignatura Lengua española III que se impartió en la Laurea in Lingue e culture per il turismo e il commercio internazionale de la Università degli Studi di Verona durante el curso 2016/2017. Dado que el alumnado adquiere de una forma más efectiva los conocimientos propuestos si los contenidos están conectados con conceptos del mundo real, se escogió como metodología, tras haber hecho una investigación previa, el Aprendizaje Basado en Proyectos (ABP), con la intención de buscar la motivación e implicación del estudiantado en su proceso de enseñanzaaprendizaje así como de lograr un aprendizaje significativo. De esta forma el alumnado estableció un manejo interdisciplinar de todas las asignaturas que había cursado en el grado, además de hacer un uso real de la lengua española a nivel profesional a través de la propuesta de proyectos de carácter turístico y/o comercial. 


\section{Palabras Clave}

Aprendizaje basado en proyectos; Lengua para fines específicos; Modos de Organización del discurso; Comercio internacional; Turismo.

\section{Abstract}

The aim of the present article is to present the results of a research and a teaching experience carried out in the subject of Spanish language III at the Laurea in Lingue and culture for tourism and international trade at the Università degli Studi in Verona during the academic year 2016/2017. Taking into account that students learn better if contents are connected to concepts of the real world, the methodology chosen, after a research process, was based on Project Based Learning (PBL). Our intention was to look for the motivation and implication of students on their own teaching-learning process. Following this methodology, students established an interdisciplinary management of all the subjects that were present in the degree and, furthermore, they also develop a real use of the Spanish language to professional level through the proposal of projects related to tourism and commerce.

\section{Key Words}

Project-Based Learning; Language for specific aims; Discourse varieties; International trade; Tourism

\section{Introducción}

La metodología del Aprendizaje Basado en Proyectos (ABP) tiene cierta tradición en enseñanzas técnicas como las ingenierías (Calvo et al., 2015), la Arquitectura o el Diseño; también en las Ciencias Sociales y, concretamente, en disciplinas como la Pedagogía (Ausín et al., 2016; Moliner y Sánchez-Tarazaga, 2015), las Ciencias de la Educación, la Música (Lasauskiene y Rauduvaite, 2015), la Educación Social (Imaz, 2015) o la Comunicación Audiovisual (Vidaurreta Ugina, 2015). No obstante, como veremos a continuación, no se ha hecho un uso tan extenso de ella en el ámbito de las Humanidades. Es posible que esta situación se deba a que el ABP tiene una visión orientada al futuro laboral del estudiantado, lo que se constata si pensamos que del empleo de esta metodología es posible obtener un "producto final", esto es, un elemento tangible, mensurable, cuantificable y, por ello, más fácilmente traducible a un código económico. 
Acaso este sea el motivo de que este tipo de metodología docente no se haya empleado de forma frecuente en el ámbito de la formación de base humanística y, concretamente, en la Lingüística, la disciplina objeto de estudio en este artículo. Esta está integrada, como se sabe, por diferentes subdisciplinas lingüísticas. Una de estas ramas sería la Lingüística aplicada, que es aquella que aplica las nociones de la Lingüística teórica a un fin concreto, sea este el de ayudar a resolver trastornos del lenguaje o el de asistir en la configuración un discurso adecuado para fines comerciales. En ella se inscribe la experiencia integrada en la asignatura a la que nos vamos a referir en lo que sigue, cuyo objetivo principal consiste en que el alumnado adquiera la capacidad de aplicar los diferentes modos de organización del discurso a los ámbitos profesionales del comercio y el turismo. Teniendo en cuenta, precisamente, tanto el objetivo de la asignatura impartida como la naturaleza económica de las disciplinas en las que se enseñaba, se creyó adecuado plantear una propuesta didáctica que incluyese una doble vertiente teórico-práctica integrada por los contenidos y su aplicación a través de la metodología que ofrece el ABP. El objetivo del presente artículo consistirá, por tanto, en la presentación de esta práctica didáctica.

\section{Contextualización}

La investigación y experiencia que se presentan en este artículo ha sido llevada a cabo en la Universitá degli Studi di Verona. Esta institución contaba, en el curso de la realización de esta investigación, con un total de 23.033 estudiantes y la Laurea in Lingue e culture per il turismo e il commercio internazionale era uno de los 63 grados ofrecidos (AA.VV; S.F.). Por su parte, la asignatura que nos interesa, Lengua española 3, pertenece al grado mencionado y se cursa en el tercer y último año. Consta de 9 créditos y equivale a 36 horas totales de actividad docente (clases, tutorías y correcciones) distribuidas en en 2 sesiones semanales de 2 horas cada una celebradas a lo largo del segundo cuatrimestre del curso que comprendía desde el 27 de febrero al 10 de junio de 2017. De cada sesión se destinaron los 20 minutos finales a trabajar por proyectos tal y como se explicará más adelante. Asimismo también se estipularon 2 sesiones de tutorías a la semana de 1 hora cada una. Es importante considerar que, para poder examinarse de esta asignatura, el alumnado debe presentar una certificación del nivel C.1. del MCER acreditado por una institución oficial. 
Como se ha adelantado, esta asignatura fue diseñada para impartir contenidos lingüísticos relacionados con tipología discursiva y pragmática desde un punto de vista eminentemente práctico; esto es, con la finalidad de aplicar transdisciplinarmente los saberes adquiridos a lo largo del grado universitario en el área del turismo y del comercio de este alumnado italiano (e italohablante) de educación superior. Con este propósito se pretendía cumplir los objetivos formativos de la asignatura. Al final de esta el alumnado: -Estará familiarizado con el español para fines específicos y con los tipos textuales habituales de la economía, el comercio y el turismo en español.

-Sabrá gestionar el uso de la lengua española en diferentes tipos de textos aplicados al comercio y al turismo.

-Conocerá diferentes realidades y contextos a la hora de analizar, seleccionar y producir discursos aplicados al español de los negocios con especial atención al comercio y al turismo.

La forma de evaluación que se preveía para esta asignatura era la realización de un examen final de la misma. Para dicho examen se matricularon 143 alumnos, aunque solo 72 pertenecían al curso escolar (2016/2017) en el que se desarrolló la experiencia y los restantes provenían de cursos pasados.

Asimismo, además del examen, se incluyó como herramienta de evaluación la posibilidad de incrementar la nota por medio de la creación de proyectos. Estos se podrían realizar de una manera totalmente voluntaria. La no realización de estos trabajos no implicaba una penalización en la nota final de la asignatura, sino que el proceso finalizado del proyecto implicaría que el alumno podría subir nota pero nunca bajarla.

Al hilo de este contexto el docente de la asignatura se propuso llevar a cabo una experiencia didáctica con los objetivos a continuación se indican.

-Enseñar al alumnado a diseñar proyectos aplicados a su realidad académico-profesional. -Diseñar un plan para poder aplicar los contenidos relacionados con Tipología discursiva, Retórica y Pragmática al contexto académico-profesional del perfil de alumnado anteriormente mencionado.

-Estimular focos de interés hacia fenómenos turístico-culturales peninsulares y latinoamericanos. 


\section{Marco teórico}

Según el Buck Institute for Education, institución especializada en la formación de profesorado en el trabajo por proyectos, el ABP es un método de enseñanza en el cual los estudiantes obtienen conocimiento y habilidades trabajando durante un extenso período de tiempo para investigar y responder a una auténtica, atrayente y compleja cuestión, problema o desafío (AA.VV, s.f.1).

El aprendizaje basado en proyectos es una metodología a la que actualmente se le asigna, en el contexto del sistema educativo español, la etiqueta de "innovación didáctica". En este sentido, el profesor de la Universidad de Granada Fernando Trujillo (2017, p. 43) acierta al denominar el tratamiento del ABP como una "innovación centenaria", puesto que este supuestamente novedoso sistema de trabajo tiene sus inicios ya en el siglo XIX de la mano del pragmático estadounidense John Dewey (1997; 2008) (Barreras, 2017, p. 114) y, posteriormente, gracias a su colega William Heart Kilpatrick quien, en 1918, establece las pinceladas básicas de la metodología de proyectos aplicados a la educación. Ambos autores defienden el valor de la experiencia en el proceso de enseñanza-aprendizaje. Si ponemos la lupa en el estado español vemos que será el inspector de educación Fernando Sáinz Ruiz quien, allá por 1928, hable por vez primera del ABP (Trujillo, 2017, p. 43).

Como se adelantó anteriormente, el trabajo por proyectos se incluye dentro del membrete "innovación educativa"; sin embargo el concepto, como hemos explicado, no es innovador. No obstante, como bien apunta Sandi Harwell (1997), es verdad que el concepto del ABP ha sido reintroducido en los ámbitos educativos en las últimas décadas. En efecto, la aplicación de esta metodología puede constituir cierta novedad si pensamos en la enseñanza universitaria tradicional (Kolmos et al., 2008, p. 9) y/o de disciplinas con gran arraigo en la educación superior. Un ejemplo a este respecto es el que constituye la enseñanza en el área de Humanidades y, más concretamente, la docencia de la Lingüística, campo en el que la vertiente teórica no siempre deja lugar al trabajo de tipo práctico. Es posible, sin embargo, que la enseñanza relacionada en concreto con la lingüística aplicada, como veremos en lo que sigue, ofrezca más libertad a la hora de introducir diferentes métodos de enseñanza como el tratado en este artículo. 
En todo caso resulta importante considerar que el ABP permite ensamblar la teoría y la práctica en la clase, así como potenciar diferentes habilidades intelectuales yendo más allá que la memorización como objetivo. Este constituye, por tanto, uno de los motivos por los que se ha escogido el ABP para el desarrollo de la experiencia didáctica que presentamos. Además, creemos que, con esta metodología, se puede promover la responsabilidad de cada individuo debido a la distribución de tareas que se realiza dentro del grupo estimulando, además, el aprendizaje colaborativo (Álvarez, Herrejón, Morelos, y Rubio, 2010, p. 2). El trabajo por proyectos implica el trabajo en grupos y esta repartición permite alcanzar aprendizajes significativos y el desarrollo de habilidades como la observación, el análisis, la negociación, la capacidad crítica o la sociabilización.

Entre las investigaciones publicados en los últimos años antes mencionadas importa recordar ahora el de Kokotsaki, Menzies, y Wiggins (2016) quienes, a partir de 53 trabajos de referencia, hacen un recorrido sobre lo escrito y teorizado sobre esta metodología. De todos ellos nos interesa en este punto el de Thomas (2000), quien propone cinco características esenciales para los proyectos (2000, pp. 3-4):

-Los proyectos obtenidos del ABP son centrales y no periféricos al currículum.

-Los proyectos obtenidos del ABP están centrados en cuestiones o problemas que conducen a los discentes a enfrentarse (e incluso a pelearse) con conceptos y principios de una disciplina

-Los proyectos involucran a los discentes en una investigación constructiva

-Los proyectos están dirigidos por los discentes hacia un nivel significativo. No tienen caminos predeterminados.

-Los proyectos son, ante todo, realistas. Esto incluye los objetivos, el tema, los papeles las tareas...

Este autor plantea que existen pruebas que acreditan que el ABP, en comparación con otros métodos de carácter instruccional, tiene un valor específico debido a que aumenta la calidad del aprendizaje por parte de los discentes en asignaturas que les pueden resultar problemáticas (Thomas, 2000, p. 35). Esto está asociado con el incremento de la capacidad por parte de estos estudiantes para aplicar aquellos elementos aprendidos en contextos en los que hay que resolver problemas. Asimismo, este investigador afirma que el ABP es un método ideal para enseñar a los estudiantes procesos complejos como la planificación, la comunicación, la resolución de problemas o la toma de decisiones. 
Es importante, por otra parte, reflexionar acerca del papel del profesor en este tipo de metodología. Efstratia (2014, p. 1258), a partir de Larmer y Mergendoller (2010) y Frey (1991), propone que el éxito del método del ABP radica en una serie de elementos clave cuya síntesis se puede encontrar en que los docentes deben imbricar los focos de interés del alumnado con las necesidades de conocer. La amalgama de estos dos elementos sería el "tópico generativo" o "pregunta conductora" (Efstratia, 2014, p. 1259), es decir, una idea que controla el génesis así como el la filosofía, el desarrollo y la ejecución del proyecto.

Para finalizar este estado de la cuestión sobre la aplicación del APB a nivel general, cabe destacar que durante la consulta de la bibliografía científica se ha echado en falta en algunos momentos un cierto consenso y homogeneización en lo que se refiere a los pasos a seguir en la aplicación de este método. Este hecho puede llegar a dificultar el reconocimiento de los diferentes pasos que habría que seguir para trabajar con proyectos en las aulas. Cuando se examina la literatura académica pertinente puede llegar a transmitir cierta falta de rigor académico motivado justamente por esta falta de unificación y estandarización en la aplicación de este método aquí trabajado y es una idea que constatan algunos autores que han estudiado el ABP aplicado a asignaturas de ciencias naturales (Edmunds et al., 2016; Cook y Weaver, 2015; Han, Yalvac, Capraro, y Capraro, 2015; Lee y Bae, 2008).

Si nos centramos ahora en la aplicación del ABP en la enseñanza de lenguas es posible constatar que el empleo de dicha metodología en este campo no supone una novedad a pesar de no estar muy extendida. Ya en 1997 Antonio Roldán realizó un inventario sobre el ABP aplicado a la docencia del inglés como lengua extranjera dentro del sistema educativo español. Otro ejemplo posterior es el trabajo de María Asunción Barreras (2017) dedicado a la enseñanza universitaria de didáctica de lengua inglesa. Por su parte, Díaz y Suñén (2013), quienes se centran en el ABP aplicado a las segundas lenguas, afirman que esta metodología se aplica con frecuencia en el ámbito de la enseñanza de segundas lenguas y quizás las primeras experiencias podrían tener su inicio en la enseñanza de la lengua inglesa como lengua extranjera. Estas mismas autoras proponen que la utilización del ABP aplicado a la enseñanza de segundas lenguas conlleva un claro beneficio. Asimismo debe recordarse la aplicación de este método en el campo de la traducción. En 
este sentido pueden recordarse los trabajos de Julia Lobato (2013) así como el de Marta García González y María Teresa Veiga Díaz (2015).

Menos son los ejemplos que encontramos si nos centramos solamente en el ABP aplicado a la docencia de la lingüística en el entorno universitario, campo en el que podemos mencionar el ejemplo de Muñoz et al., (2013), quienes exponen los resultados de la Red de Diseño e Implementación de Materiales Docentes con ABP en un trabajo en el que exploran las aplicaciones del ABP a la enseñanza de la asignatura Lingüística general I en la Universidad de Alicante durante el curso escolar 2010-2011. Estos autores exponen su experiencia didáctica con 72 alumnos y 13 proyectos presentados (Muñoz et al., 2013, p. 1692).

\section{Experiencia didáctica}

\subsection{Contenidos}

Para la configuración y selección de los contenidos de lingüística aplicada que se impartieron en esta asignatura, y dadas las necesidades laborales futuras del alumnado, se escogieron conocimientos pertenecientes a los diferentes modos de organización del discurso así como de Retórica y Pragmática. Estas disciplinas o subdisciplinas se plantearon de una manera interconectada y con un carácter eminentemente práctico para alcanzar los objetivos que tendría que cumplir el alumnado de la materia. Los contenidos propuestos para esta asignatura y la secuenciación de los mismos fue la que se puede observar a continuación:

\begin{tabular}{|c|c|c|}
\hline $\begin{array}{c}\text { Número } \\
\text { de } \\
\text { sesión }\end{array}$ & Fecha & Contenido de la materia \\
\hline 1 & 28 de febrero & $\begin{array}{r}\text { Sesión presentación } \\
\text { 1. Destreza discursiva (Tipología discursiva) y } \\
\text { Destreza Pragmática - Proyectos }\end{array}$ \\
\hline
\end{tabular}




\begin{tabular}{|c|c|c|}
\hline 2 & 1 de marzo & $\begin{array}{l}\text { Contextualización de la materia } \\
\text { La comunicación } \\
\text { La competencia comunicativa } \\
\text { Las lenguas con fines específicos } \\
\text { El español del turismo }\end{array}$ \\
\hline 3 & 7 de marzo & $\begin{array}{c}\text { Tipología discursiva: } \\
\text { Los textos como unidades comunicativas } \\
\text { El discurso } \\
\text { Discurso oral/ Discurso escrito } \\
\text { Análisis del discurso }\end{array}$ \\
\hline 4 & 8 de marzo & Tipología discursiva: El texto narrativo \\
\hline 5 & 14 de marzo & Tipología discursiva: El texto descriptivo \\
\hline 6 & 15 de marzo & Tipología discursiva: El texto expositivo \\
\hline 7 & 21 de marzo & Tipología discursiva: El texto expositivo \\
\hline 8 & 22 de marzo & $\begin{array}{c}\text { Tipología discursiva: El texto argumentativo - La teoría } \\
\text { de la argumentación (Anscombre y Ducrot) }\end{array}$ \\
\hline 9 & 28 de marzo & $\begin{array}{c}\text { Tipología discursiva: El texto argumentativo - } \\
\text { Argumentación publicitaria }\end{array}$ \\
\hline 10 & 29 de marzo & $\begin{array}{l}\text { Textos legales: Legislación sobre el turismo en España } \\
\qquad \text { - Contratos }\end{array}$ \\
\hline 11 & 11 de abril & $\begin{array}{c}\text { Textos formales (Instancias, recursos, peticiones) y } \\
\text { textos comerciales. }\end{array}$ \\
\hline 12 & 12 de abril & Procedimientos retóricos para la creación de discursos \\
\hline 13 & 19 de abril & $\begin{array}{c}\text { Introducción a la Pragmática } \\
\text { El contexto (SPEAKING) - El contexto cultural } \\
\text { compartido } \\
\text { Los fines discursivos (metas y productos) }\end{array}$ \\
\hline 14 & 26 de abril & Austin y Searle: Los actos de habla \\
\hline 15 & 2 de mayo & Grice y el principio de cooperación \\
\hline
\end{tabular}




\begin{tabular}{|c|c|c|}
\hline 16 & 3 de mayo & La teoría de la Relevancia (Sperber y Wilson) \\
\hline 17 & 9 de mayo & La cortesía lingüística \\
\hline 18 & 10 de mayo & Clase repaso y recopilación \\
\hline & 21 de junio & Entrega de los proyectos \\
\hline & 21 de junio & Examen Ordinaria \\
\hline & 6 de julio & Examen Extraordinaria \\
\hline
\end{tabular}

Tabla 1: Cronograma de impartición de los contenidos. Fuente: Elaboración propia.

\subsection{Metodología, desarrollo y evaluación}

En el proceso de organización y ejecución de la experiencia didáctica que presentamos es posible distinguir tres fases que hemos denominado preproducción, producción y postproducción y que a continuación describiremos. En la primera parte, la de preproducción, se planteará cuál fue el trabajo previo de preparación de las clases así como el acopio de materiales y recursos relativos a los proyectos que, más tarde, se tuvieron en cuenta durante el ejercicio de la docencia en Verona. En la segunda fase, la de producción, se expondrá el cronograma seguido para la impartición de esta asignatura; en él pueden apreciarse tanto los contenidos como el avance y las diferentes fases del trabajo por proyectos. Por último, en la tercera parte, la de postproducción, se explicarán los resultados del aprendizaje así como los criterios utilizados para la evaluación de los proyectos.

\subsubsection{Preproducción}

De forma previa al diseño de la asignatura se plantea la posibilidad de trabajar con el alumnado dos tipos de variantes del español. Por un lado la variante peninsular y, por otra, la variante ecuatoriana. Se escogieron estas dos variantes para poder brindar al alumnado un ejemplo concreto del español peninsular y un ejemplo concreto de lo (inexactamente) denominado español de América pero aplicado a un entorno geográfico 
un poco más acotado. Para trabajar la variante del español de Ecuador se propone el marco temático de "La Romería de El Cisne", una peregrinación de carácter religioso que se desarrolla en la comarca de El Cisne en la provincia de Loja. Para operar con la otra variante propuesta se escoge el marco temático del Camino de Santiago. Ambos escenarios servían tanto para la contextualización de los proyectos como para la de los contenidos.

En esta primera fase resulta significativo, asimismo, hacer una breve referencia tanto a las personas con las que se estableció contacto como a la preparación del material didáctico. A este respecto, el docente recopiló documentación oficial relacionada con el impacto socioeconómico y cultural de estos fenómenos turísticos con la finalidad de establecer la posibilidad de trabajar con el alumnado diversos modos de organización del discurso en español a través de material real. Con el fin de que los estudiantes que quisieran optar por trabajar con el marco temático de La Virgen de El Cisne pudieran contar con ayuda académica especialista en este tema, el docente de esta asignatura se puso en contacto con profesorado especialista en comunicación turística de la Universidad Técnica Particular de Loja (UTPL). Por otra parte, el docente responsable de esta experiencia se puso en contacto con la entidad Sociedade Anónima de Xestión do Plan Xacobeo, la empresa pública responsable, entre otras cosas, de la difusión del Camino de Santiago, para solicitar material que sería usado como recurso educativo en este contexto. Esta entidad aportó a la Universidad de Verona los siguientes elementos:

- 50 libros

- 9 DVDs

- 600 folletos informativos sobre los diferentes caminos

- 300 guías

- 200 lápices

- 50 camisetas

\subsubsection{Producción}

Llegados a este punto, la primera cuestión relevante es llamar la atención sobre la ausencia de bibliografía con una base científica sólida sobre las fases que habría que seguir al aplicar en el aula el ABP. En este sentido se evaluaron varias propuestas 
comenzando por Kilpatrick (1929, p. 17) quien propone cuatro fases: Propósito/objetivos, Planificar, Ejecutar y Juzgar/Evaluar. Posteriormente se ha reflexionado acerca de los planteamientos recogidas en el Buck Institute for Education (S.F.) y, asimismo, en los trabajos de Blumenfeld et al. (1991), Han y Bhattacharya (2001), Tippelt y Lindemann (2001) y Pozuelos (2007); pero no se encontró en ellos una secuenciación de fases homogénea y estandarizada. Por este motivo se ha optado por unir los puntos comunes de las fases propuestas por estos autores y concentrarlas en los siguientes pasos que, estimamos, se ajustan bastante a lo propuesto originalmente por Kilpatrick: Idear, Elegir, Planificar, Realizar/Ejecutar y Revisar/Evaluar. A diferencia de lo establecido por el pedagogo norteamericano se opta por dividir su fase Propósito/objetivos (Purpouses) en dos, ya que de esta manera se pueden efectuar dinámicas de creatividad para originar ideas (Idear) y así, posteriormente, poder seleccionar (Elegir) la más adecuada a los objetivos que se presentan en el proyecto propuesto por el alumnado.

El 28 de febrero de 2017, primera clase, se presenta la asignatura al alumnado. Además de dar a conocer los contenidos, se le ofrece a los discentes la oportunidad de realizar un proyecto de carácter voluntario con la posibilidad de que este pueda ayudar a subir la nota final de la asignatura. En esta primera sesión se explican los diferentes procedimientos a seguir con los proyectos y cuáles serán las fases del mismo. Uno de estos procedimientos es la libre formación de grupos configurados por conjuntos de entre 4 y 7 miembros. Cada grupo debe tener un nombre singular y bien argumentado. Se les ofrece unas breves directrices sobre cómo se idea y se escoge un nombre para una entidad. Asimismo, se adelanta a los estudiantes que cada uno tendrá una tarea definida en el grupo. Esta acción no es baladí, puesto que pone a prueba la autoevaluación del estudiante haciéndose que se pregunte “¿Qué sé hacer?” y “¿Qué me gustaría hacer?” dentro de este proyecto. De igual manera también se les anticipa que deberán elegir a una persona delegada que será la que tenga comunicación directa y fluida con el docente de la asignatura para solucionar eventualidades derivadas de esta experiencia.

Dicho esto se le explica al alumnado la primera fase, la correspondiente a idear, y se invita a que cada grupo aporte una media de cuatro ideas. Para esto, se adelanta que, en la siguiente sesión, se realizará una dinámica básica sobre generación de ideas. En esta primera fase se plantea la idea controladora o tópico generativo que el alumnado debe seguir que es el siguiente: ¿Qué necesidades se pueden cubrir desde el punto de vista 
del comercio y del turismo y mediante un proyecto en el camino de Santiago o en la romería de El Cisne en Loja?

Debido a la naturaleza profesionalizante de este grado universitario y, ya que las personas involucradas nunca habían diseñado un proyecto, se les propone la posibilidad de realizar dos tipos de productos finales. El primero implicaría la ejecución del proyecto diseñado antes del 21 de junio (fecha de entrega de proyectos), a lo que denominamos "proyecto profesional". El segundo supondría la entrega de un plan de viabilidad del proyecto, esto es, una guía detallada en la cual se explicaría punto por punto cómo debería planificarse y ejecutarse el proyecto en otro momento diferente al periodo escolar. A este último tipo lo denominaremos "proyecto académico".

En esta fase inicial apreciamos una variable que no se había contemplado hasta el momento: la rica multiculturalidad que habita en Verona, su universidad y en el grupo en el que se ha llevado a cabo esta experiencia. Como se expuso anteriormente en este artículo, el tópico generativo que se había propuesto estaba relacionado con dos fenómenos turísticos religiosos de base cristiana lo que, dada la multiculturalidad veronesa, podría haber causado algún tipo de conflicto en otras comunidades religiosas a las que pertenecían algunos estudiantes del grupo con el que se estuvo trabajando. Detectado este hecho, el docente optó por dialogar con las personas que ostentaban signos de religiones diferentes a las de base cristiana, con el fin de verificar si le resultaba un tópico generativo acorde con sus valores o si, por el contrario, era necesario buscar otros posibles focos de interés para estos discentes. El alumnado afirmó estar de acuerdo con este tipo de metodología así como con los dos temas principales, aunque finalmente no participaron en esta experiencia con ABP. En lo que se refiere al trabajo basado en proyectos con grupos heterogéneos de estudiantes merece la pena recordar las ideas de Voronchenko, Klimenko, y Kostina (2014). Estas autoras ponen de manifiesto el grado de tolerancia que el alumnado que trabaja con ABP puede adquirir, ya que la tarea colaborativa intrínseca a este método mejora la cultura de la tolerancia, el respeto a posiciones diferentes así como el entendimiento ante distintos fenómenos sociales (Voronchenko, Klimenko, y Kostina, 2014, p. 1494).

El día 1 de marzo se propone la creación de grupos de trabajo a los que deberán otorgar un nombre. Asimismo se plantean algunas dinámicas de generación de ideas para conocer procesos sobre cómo idear proyectos. 
El día 8 de marzo se ponen en funcionamiento los proyectos con 77 alumnos divididos en 15 grupos. Esto indica que 5 de los alumnos que deciden participar en la experiencia provenían de cursos pasados. 12 grupos trabajarán con el Camino de Santiago y 3 trabajarán sobre la romería de El Cisne.

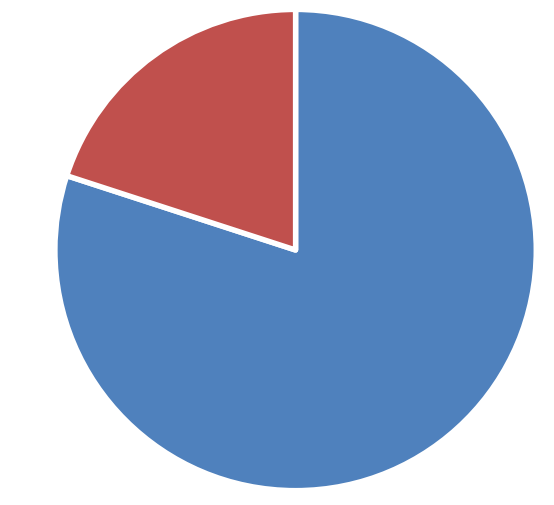

- Camino de Santiago - Romería del Cisne

Gráfico 1: Visualización de los ejes temáticos escogidos para los proyectos. Fuente: Elaboración propia.

Ante la inexperiencia del alumnado en la aplicación a la realidad de los conocimientos obtenidos en su formación universitaria, estos optan por escoger la modalidad de "proyecto académico" frente al "proyecto profesional".

Parece significativo apuntar que en el grupo general se constata una falta de destrezas para hablar en público; sin embargo mostraba cierta confianza y fluidez cuando acudía a las tutorías. Este tipo de atención al alumnado se convirtió en una de las herramientas más efectivas a la hora de completar la formación en dinámicas de proyectos, ya que durante la celebración de las mismas los discentes planteaban todo tipo de cuestiones pertinentes que, por miedo o por vergüenza (parámetros que nombraron varios alumnos), no hacían en clase delante de sus compañeros. En definitiva, las tutorías han resultado cruciales para ayudar al alumnado a repartirse las tareas en el grupo y, también, para que cada persona desempeñara un trabajo acorde con sus conocimientos y deseos. Creemos relevante apuntar que durante las tutorías el alumnado tenía que hablar en lengua castellana, lo que provocaba una situación real en la que los estudiantes tenían que exponer, explicar y argumentar oralmente en lengua castellana. Se constata, de esta 
forma, la relevancia de la tutoría en la educación superior tratada por diversos autores (López Gómez, 2017) y, de manera concreta, su utilidad en el desarrollo de la metodología empleada.

Con todo lo expuesto hasta el momento, mostramos a continuación el cronograma que se siguió en el cual se indica el número de sesión, la fecha de la sesión, el contenido impartido en la sesión así como la fase de los proyectos tratada en dicha sesión:

\begin{tabular}{|c|c|c|c|}
\hline $\begin{array}{l}\text { Número } \\
\text { de } \\
\text { sesión }\end{array}$ & $\begin{array}{l}\text { Fech } \\
\text { a }\end{array}$ & Contenido de la materia & $\begin{array}{l}\text { Fase del } \\
\text { proyecto }\end{array}$ \\
\hline 1 & $\begin{array}{l}28 \mathrm{de} \\
\text { febrer } \\
0\end{array}$ & $\begin{array}{c}\text { Sesión presentación - Destreza discursiva } \\
\text { (Tipología discursiva) y Destreza } \\
\text { Pragmática - Proyectos }\end{array}$ & $\begin{array}{l}\text { Explicación } \\
\text { de lo que es } \\
\text { un proyecto }\end{array}$ \\
\hline 2 & $\begin{array}{c}1 \mathrm{de} \\
\operatorname{marz} \\
0\end{array}$ & $\begin{array}{c}\text { Contextualización de la materia } \\
2.1 \text { La comunicación } \\
\text { 2.2 La competencia comunicativa } \\
\text { 2.3 Las lenguas con fines específicos } \\
2.5 \text { El español del turismo }\end{array}$ & $\begin{array}{l}\text { Creación de } \\
\text { grupos e } \\
\text { Idear } \\
\text { proyecto }\end{array}$ \\
\hline 3 & $\begin{array}{l}7 \mathrm{de} \\
\text { marz } \\
0\end{array}$ & $\begin{array}{c}\text { Tipología discursiva: } \\
\text { 3.1 Los textos como unidades } \\
\text { comunicativas } \\
\text { 3.2 El discurso } \\
\text { 3.3 Discurso oral/ Discurso escrito } \\
\text { 3.4 Análisis del discurso }\end{array}$ & $\begin{array}{c}\text { Idear } \\
\text { proyecto }\end{array}$ \\
\hline 4 & $\begin{array}{c}8 \mathrm{de} \\
\text { marz } \\
0\end{array}$ & Tipología discursiva: El texto narrativo & Elegir \\
\hline 5 & $\begin{array}{l}14 \text { de } \\
\text { marz } \\
0\end{array}$ & Tipología discursiva: El texto descriptivo & Elegir \\
\hline 6 & $\begin{array}{l}15 \mathrm{de} \\
\text { marz } \\
0\end{array}$ & Tipología discursiva: El texto expositivo & Planificar \\
\hline 7 & $\begin{array}{l}21 \mathrm{de} \\
\text { marz } \\
0\end{array}$ & Tipología discursiva: El texto expositivo & Planificar \\
\hline 8 & $\begin{array}{c}22 \mathrm{de} \\
\text { marz } \\
0\end{array}$ & $\begin{array}{l}\text { Tipología discursiva: El texto argumentativo } \\
\text { - La teoría de la argumentación (Anscombre } \\
\text { y Ducrot) }\end{array}$ & Planificar \\
\hline 9 & $\begin{array}{c}28 \mathrm{de} \\
\text { marz } \\
0\end{array}$ & $\begin{array}{l}\text { Tipología discursiva: El texto argumentativo } \\
\text { - Argumentación publicitaria }\end{array}$ & Planificar \\
\hline
\end{tabular}




\begin{tabular}{|c|c|c|c|}
\hline 10 & $\begin{array}{c}29 \text { de } \\
\text { marz } \\
0\end{array}$ & $\begin{array}{l}\text { Textos legales: Legislación sobre el turismo } \\
\text { en España - Contratos }\end{array}$ & Planificar \\
\hline 11 & $\begin{array}{l}11 \text { de } \\
\text { abril }\end{array}$ & $\begin{array}{l}\text { Textos formales (Instancias, recursos, } \\
\text { peticiones) y textos comerciales. }\end{array}$ & Planificar \\
\hline 12 & $\begin{array}{l}12 \text { de } \\
\text { abril }\end{array}$ & $\begin{array}{c}\text { Procedimientos retóricos para la creación } \\
\text { de discursos }\end{array}$ & Planificar \\
\hline 13 & $\begin{array}{l}19 \text { de } \\
\text { abril }\end{array}$ & $\begin{array}{c}\text { Introducción a la Pragmática } \\
\text { El contexto (SPEAKING) - El contexto } \\
\text { cultural compartido } \\
\text { Los fines discursivos (metas y productos) }\end{array}$ & Planificar \\
\hline 14 & $\begin{array}{l}26 \text { de } \\
\text { abril }\end{array}$ & Austin y Searle: Los actos de habla & Planificar \\
\hline 15 & $\begin{array}{l}2 \text { de } \\
\text { mayo }\end{array}$ & Grice y el principio de cooperación & Planificar \\
\hline 16 & $\begin{array}{l}3 \text { de } \\
\text { mayo }\end{array}$ & $\begin{array}{c}\text { La teoría de la Relevancia (Sperber y } \\
\text { Wilson) }\end{array}$ & Planificar \\
\hline 17 & $\begin{array}{l}9 \mathrm{de} \\
\text { mayo }\end{array}$ & La cortesía lingüística & Planificar \\
\hline \multirow[t]{4}{*}{18} & $\begin{array}{l}10 \text { de } \\
\text { mayo }\end{array}$ & Clase Repaso y recopilación & Planificar \\
\hline & $\begin{array}{l}21 \text { de } \\
\text { junio }\end{array}$ & Entrega de los proyectos & Realizar \\
\hline & $\begin{array}{l}21 \text { de } \\
\text { junio }\end{array}$ & Examen Ordinaria & \\
\hline & $\begin{array}{l}6 \text { de } \\
\text { julio }\end{array}$ & Examen Extraordinaria & \\
\hline
\end{tabular}

Tabla 2: Cronograma de la experiencia didáctica. Fuente: Elaboración propia.

Resulta relevante señalar, en lo que concierne al desarrollo de esta fase, que en las sesiones 11 y 12 se aprecia un descenso de la motivación del alumnado a la hora de continuar con sus proyectos. En estas fechas se comienza a hablar de los exámenes y trabajos de otras asignaturas y el proyecto, según exponen algunos alumnos en tutorías, se ve como algo prescindible debido a su carácter voluntario. Ante este hecho el docente hubo de buscar posibles estrategias de motivación y, finalmente, optó por la siguiente. Decide hablar con una antigua alumna de Comunicación Audiovisual de la Universidad de Santiago de Compostela, que había conseguido llevar a cabo su proyecto 
satisfactoriamente, y pedirle que haga un vídeo de carácter inspirador en donde se viera que el diseño y ejecución de un proyecto es algo factible e ilusionante. Dicho vídeo se recibió el 10 de abril y se proyectó el 12 de abril. Esto consiguió una "reactivación" de los proyectos de los estudiantes que siguieron adelante con la realización de sus proyectos.

\subsubsection{Postproducción}

A día 21 de junio 44 alumnos, de los que 40 eran mujeres y 4 hombres, entregaron 10 proyectos de los 15 propuestos inicialmente. Del número total de proyectos, 3 versaron sobre el Camino de Santiago, 3 sobre la romería de El Cisne y 4 sobre temática libre escogida por el alumnado. En este sentido importa señalar el carácter motivador de este tipo de metodología de trabajo. Así pues, de los 12 proyectos inicialmente vinculados con el Camino de Santiago, 3 versaron finalmente sobre temas derivados de este tópico generativo aunque no propiamente con él. Esto significa, pues, que el alumnado implicado logró idear un nuevo tema por sí mismo gracias al tópico primero.

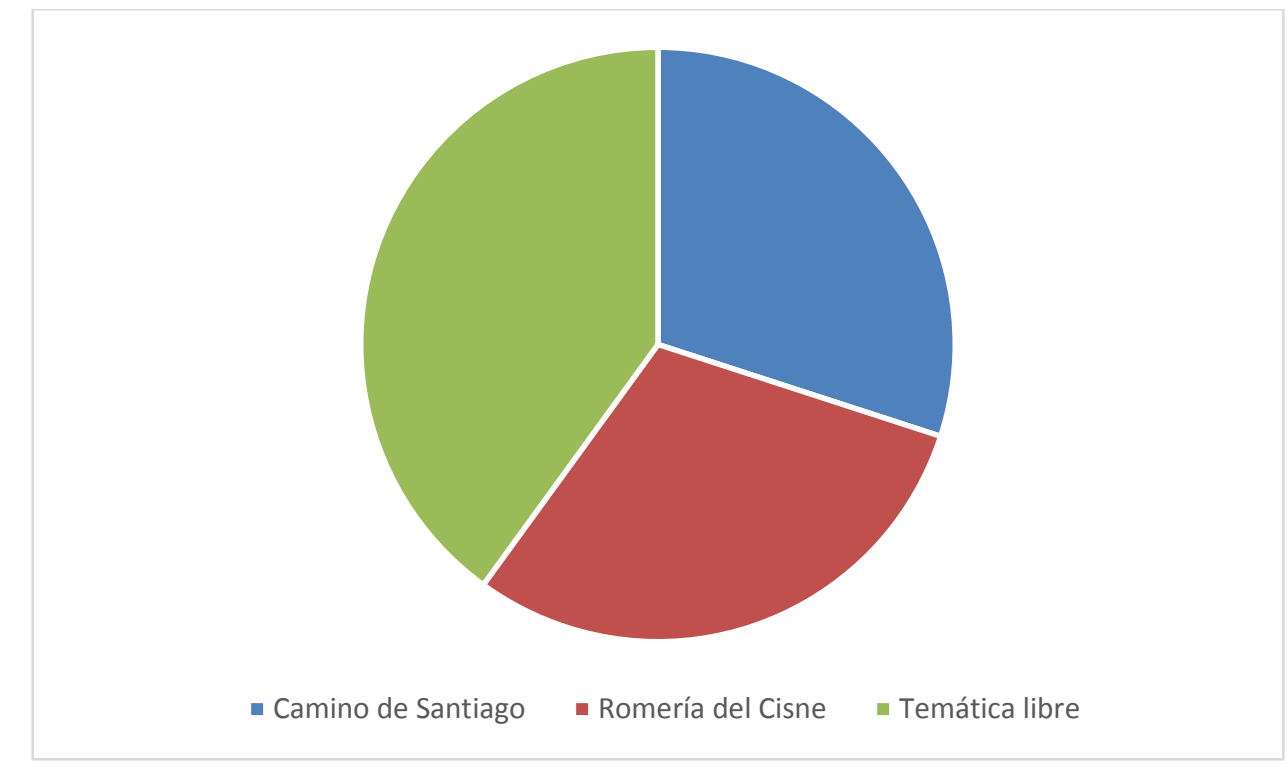

Gráfico 2: Visualización de los ejes temáticos escogidos finalmente para los proyectos. Fuente: Elaboración propia.

Si nos fijamos en el número de alumnos podemos concluir que un $57 \%$ del porcentaje total que comenzó la experiencia la ha concluido con la presentación del proyecto; mientras que si prestamos atención a los proyectos, podemos señalar que un $66,6 \%$ de los proyectos inicialmente presentados han sido concluidos. 
Para evaluar los proyectos entregados por el alumnado se han utilizado los siguientes criterios. El proyecto entregado al docente debe:

- Ser original.

- Tener bien definido el público objetivo al que va dirigido el proyecto.

- Tener una cierta viabilidad o aplicación real.

- Demostrar con claridad la forma de obtener datos para la realización del proyecto.

- Reflejar las tareas realizadas por cada uno de los miembros del grupo.

- Demostrar un nivel de competencia de la lengua castellana equivalente a un C.1. por el MCER.

- Demostrar que las narraciones, descripciones, exposiciones y argumentaciones incluidas en los proyectos están bien estructuradas y presentan coherencia, cohesión y adecuación.

Una vez evaluados estos proyectos se pasó a la calificación; los 10 entregados obtuvieron de media un 27,1 sobre 30 , siendo 24 la calificación más baja y 30 la más alta. Dicha escala numérica es la que se utiliza en educación dentro del estado italiano. Estas calificaciones pasarían a hacer media con las notas obtenidas por el alumnado en su examen final, siempre y cuando estos lo escogieran así.

De los 44 alumnos que concluyeron la experiencia 24 se presentaron al examen de esta asignatura. De estos 24 alumnos, 15 aprobaron la asignatura en la $1^{\underline{a}}$ convocatoria, 7 aprobaron en $2^{\mathrm{a}}$ convocatoria y 2 suspendieron la asignatura. Por lo tanto, nos encontramos ante un porcentaje de un $82 \%$ de alumnos que han superado la materia con respecto al total de alumnos que se han presentado al examen.

\section{Conclusiones}

La investigación efectuada para el trazado del marco teórico y la posterior experiencia didáctica realizada nos ha permitido establecer las conclusiones que a continuación se ofrecen.

En primer debemos considerar que, a través de estos proyectos, el alumnado ha logrado contestar a la pregunta conductora relativa a las ausencias de negocio observadas en el 
Camino de Santiago y en la Romería del Cisne, que constituía un problema o desafío auténtico y atrayente si consideramos su campo de especialización y, por tanto, su posible ámbito laboral relacionado con el comercio internacional y el turismo. Esto supone, además, que el alumnado podrá aplicar lo aprendido en esta materia en contextos laborales futuros en los que haya que resolver problemas de igual naturaleza. Asimismo, se ha promovido la responsabilidad de cada miembro a través de la distribución de tareas aunque se ha estimulado, también, la colaboración. A lo largo del período de realización de los proyectos cada uno de los grupos ha observado las posibles ideas con las que contaban y ha negociado para la elección de la idea argumentando a favor o en contra de ella lo que, inevitablemente, favorece la sociabilización en el grupo.

En segundo lugar es importante tener en cuenta cómo, a través del ABP, hemos planteado una asignatura en la que se ensambla teoría y práctica, esto es, los contenidos lingüísticos señalados y su ejercicio en el contexto de los proyectos, tanto en el producto final como a través de su fase de preparación, período durante el cual el alumnado se ha visto en la obligación de ejercitar la competencia comunicativa por medio de las siguientes tareas:

- Búsqueda de información relacionada con el tema del proyecto de cada grupo en lengua castellana.

- Conversaciones transaccionales por medio de videoconferencias con personas hispanohablantes con el objetivo de obtener información para su proyecto.

- Intercambio de correos electrónicos de carácter transaccional con personas hispanohablantes con el objetivo de obtener información para su proyecto.

Más allá de los contenidos de la asignatura que nos compete, el ABP nos ha permitido también que el alumnado hiciera uso en los proyectos de los conocimientos adquiridos en otras asignaturas del grado de forma integradora. En este sentido podemos mencionar, por ejemplo, la Informática, los Fundamentos de gestión, la Sociología del proceso cultural, la Teoría y técnica de la comunicación, la Historia del turismo, el Marketing internacional o el Marketing turístico para crear un proyecto real, un proyecto con el que podrían iniciar una carrera laboral o un proyecto que podría dar lugar a otros más maduros. 
No podemos olvidar que el pilar fundamental, el elemento cohesionador y estructurador de todos los conocimientos aplicados en los proyectos ha sido la lengua española y sus diferentes modos de organización y adecuación del discurso vehiculizado por la tipología discursiva, la Retórica y la Pragmática. Por una parte, importa considerar que el alumnado se tuvo que documentar con fuentes en español sobre los temas escogidos así como acerca del público objetivo al que iba dirigido su propuesta, con lo que se trabajó la comprensión de lectura. En 6 de estos proyectos los grupos tuvieron que intercambiar correos y hacer videoconferencias con fuentes hispanohablantes tales como recepcionistas de hostales de España o profesores universitarios de Ecuador, lo que también suponía ejercitar los contenidos del temario y trabajar, asimismo, la comprensión auditiva, la comprensión de lectura, la interacción oral y la expresión oral. Asimismo cabe destacar que, para la ejecución de los proyectos, este estudiantado italiano tuvo que describir, narrar, exponer y argumentar en lengua castellana, lo que implicó poner en práctica todos los contenidos teóricos sobre tipología discursiva, Retórica y Pragmática impartidos y, asimismo, la expresión escrita. Podemos constatar, por lo tanto, que se han cumplido los objetivos de la asignatura. Esta idea puede reforzarse con los resultados obtenidos en el examen por parte del alumnado que había participado en la experiencia de proyectos, puesto que, del total de 24, 22 aprobaron la materia.

Resulta relevante señalar cómo, progresivamente, se observó una implicación del alumnado en su propio proceso de enseñanza-aprendizaje tanto a nivel formal (modos de organización del discurso en lengua española) como a nivel de contenidos (los temas turísticos propuestos para la realización de esta experiencia didáctica). En relación con la última idea, podemos afirmar que se han estimulado los focos de interés hacia fenómenos turístico-culturales peninsulares y latinoamericanos fuera de los establecidos canónicamente. Prueba de ello son los 6 proyectos entregados cuya temática estaba relacionada con el Camino de Santiago o con la romería de El Cisne. Es necesario añadir, asimismo, que uno de los proyectos relacionados con el fenómeno turístico-religioso ecuatoriano derivó en un trabajo de fin de grado.

Por medio de la observación en las tutorías, de los proyectos y de los exámenes se ha comprobado una más que adecuada fijación de los contenidos propuestos para esta asignatura, por lo que estimamos que el ABP resulta un método muy útil para este tipo de 
asignaturas de base lingüística en un grado relacionado con el comercio y el turismo, ya que otorga un uso aplicado y práctico de la lingüística para un entorno profesional. Por último, y como uno de los posibles defectos achacables a esta experiencia, debe considerarse que se ha echado en falta, y se tendrá en cuenta para el futuro, la presencia de medición en el progreso de la adquisición de contenidos por parte del alumnado; ya que estimamos que solamente la observación directa del docente, los exámenes y los proyectos concluidos pueden resultar insuficientes para apreciar una evolución gradual.

\section{Bibliografía}

\section{PUBLICACIONES PERIÓDICAS}

Álvarez, V., Herrejón, V.D.C., Morelos, M. y Rubio, M.T., (2010). Trabajo por proyectos: aprendizaje con sentido. En Revista Iberoamericana de Educación / Revista Ibero-americana de Educação. 52/5, 1-13.

Ausín, V., Abella, V., Delgado, V., y Hortigüela, D. (2016). Aprendizaje Basado en Proyectos a través de las TIC. Una Experiencia de Innovación Docente desde las Aulas Universitarias. Formación Universitaria, 9(3). DOI: 10.4067/S071850062016000300005 .

Barreras, M. A. (2017). Modelo PBL en la docencia universitaria de la didáctica de la lengua inglesa. Tejuelo, 25, 111-144. DOI: 10.17398/1988-8430.25.111

Blumenfeld, P. C., Soloway, E., Marx, R., Krajcik, J., Guzdial, M., y Palincsar, A. (1991). Motivating project-based learning: Sustaining the doing, supporting the learning. Educational Psychologist, 26(3 \& 4), 369-398.

Calvo, I. Quesada, J. Olalde, K y García, B. (2015). Experiencias docentes de la aplicación de PBL en Ingeniería. Ikastorratza e-Revista de didáctica, 14(4), 39-71. 
Cook, N. D., y Weaver, G. C. (2015). Teachers' implementation of project-based learning: Lessons from the research goes to school program. Electronic Journal of Science Education, 19(6).

Díaz, E y Suñén, M. C, (2013). El trabajo basado en proyectos en la clase de español con fines profesionales. Revista Nebrija de Lingüística Aplicada a la Enseñanza de Lenguas, 13.

Edmunds, J., Arshavsky, N., Glennie, E., Charles, K., y Rice, O. (2016). The Relationship between Project-Based Learning and Rigor in STEM-Focused High Schools. Interdisciplinary Journal of Problem-Based Learning, 11(1). DOI: http://dx.doi.org/10.7771/15415015.1618

Efstratia, D. (2014). Experiential education through project based learning. Procedia - Social and Behavioral Sciences, 152, 1256-1260.

Han, S., Yalvac, B., Capraro, M. M., y Capraro, R. M. (2015). Inservice teachers' implementation and understanding of STEM project based learning. Eurasia Journal of Mathematics, Science and Technology Education, 11(1), 63-76. DOI: https://doi.org/10.12973/eurasia.2015.1306a

Harwell, S. (1997). Project-based learning. En W.E. Blank \& S. Harwell (Eds.), Promising practices for connecting high school to the real world. (pp. 23-28). Tampa, FL: University of South Florida.

Imaz, J. I. (2015). Aprendizaje basado en proyectos en los grados de Pedagogía y Educación Social: ¿Cómo ha cambiado tu ciudad?. Revista Complutense de Educación, 26, 679696.

Kokotsaki, D., Menzies, V. y Wiggins, A. (2016). Project-based learning: a review of the literature. En Improving schools, 19(3), 267-277.

Kolmos, A., Du, X., Holgaard, J. E., \& Jensen, L. P. (2008). Facilitation in a PBL environment. Aalborg: UCPBL UNESCO Chair in Problem Based Learning. 
Larmer, J. y Mergendoller, J. R. (2010). Seven Essentials for Project-Based Learning. Educational Leadership, 68(1), 34-37.

Lasauskiene, J. y Rauduvaite, A. (2015). Project-Based Learning at University: Teaching Experiences of Lecturers, Procedia - Social and Behavioral Sciences, 197, 788- 792. DOI: https://doi.org/10.1016/j.sbspro.2015.07.182

Lee, H., y Bae, S. (2008). Issues in implementing a structured problem-based learning strategy in a volcano unit: A case study. International Journal of Science and Mathematics Education, 6, 655-676. DOI: https://doi.org/10.1007/s10763-007-9067-x

Lobato, J. (2013). Propuesta didáctica para las clases de traducción especializada: el aprendizaje basado en proyectos. Tonos Digital, 25(0).

López Gómez, Ernesto, El concepto y las finalidades de la tutoría universitaria: una consulta a expertos, Revista Española de Orientación y Psicopedagogía, Vol. 28, №2, 2017, pp. 61-78.

García, M.y Veiga, M.T. (2015). Guided Inquiry and Project-Based Learning in the field of specialised translation: a description of two learning experiences. Perspectives, 23(1), 107-123, DOI: 10.1080/0907676X.2014.948018

Mergendoller, J. R. y Thomas, J. W. (S.F.). Managing Project Based Learning: Principles from the Field. https://goo.gl/j4P3MM

Moliner, O. y Sánchez-Tarazaga, L. (2015). PBL o aprendizaje basado en proyectos: una experiencia de coordinación y enseñanza auténtica en la universidad. Quaderns digitals, 81, 116-133.

Roldán, A.R. (1997). El trabajo por proyectos en el sistema educativo español: revisión y propuestas de realización. Encuentro. Revista de Investigación e Innovación en la clase de idiomas, 9, 115-125.

Trujillo, F. (2017). Aprendizaje basado en proyectos. Líneas de avance para una innovación centenaria. Textos. Didáctica de la Lengua y de la Literatura, 78, 42-48. 
Voronchenkoa, T., Klimenkob, T., Kostinab, I. (2014). Learning to live in a global world: ProjectBased Learning in multicultural student groups as a pedagogy of tolerance strategy. Procedia - Social and Behavioral Sciences, 191, 1489 - 1495.

\section{LIBROS Y CAPÍTULOS DE LIBRO}

Dewey, J. (1997). Democracia y educación: una introducción a la filosofía de la educación. Madrid: Ediciones Morata.

Dewey, J. (2008). Experience and Education. New York: Touchstone.

Frey, K. (1991). Die Projectmethode. Berlin: BeltzWeinheim.

Han, S. y Bhattacharya, K. (2001). Constructionism, Learning by Design, and Project-based Learning. En M. Orey (Ed.), Emerging perspectives on learning, teaching, and technology. Georgia: Universidad de Georgia.

Kilpatrick, W. H. (1929). The project method. The use of the purposeful act in the educative process. Nueva York: Universidad de Columbia.

Muñoz, I. M., La Parra, D., Ortiz, R., Davó, M.C. y Álvarez, J.S. (2013). ReDIMABP - Red de Diseño e Implementación de Materiales Docentes con ABP (Aprendizaje Basado en Proyectos). En Álvarez, J. D., Tortosa, M. T. y Pellín, N. (Ed.s). Diseño de acciones de investigación en docencia universitaria (pp. 1684-1703). Alicante: Universidad.

Pozuelos, F. J. (2007). Trabajo por proyectos en el aula: Descripción, investigación y experiencias. Morón de la Frontera: M.C.E.P.

Thomas, J.W. (2000). A review of research on project-based learning. California: The Autodesk Foundation.

Tippelt, R. y Lindemann, H. (2001). El método de proyectos. El Salvador, München, Berlin: Unión Europea. 
Vidaurreta Ugina, Javier (2015). Implementación de metodologías activas en la docencia universitaria en Comunicación Audiovisual aplicación docente de la realización de un informativo de televisión. Madrid: Universidad Europea de Madrid. Tesis doctoral inédita.

\section{MEDIOS ELECTRÓNICOS}

AA.VV. (S.F.). 1. What is Project Based Learning (PBL)? Recuperado de http://www.bie.org/about/what_pbl.

AA.VV. (S.F.). 2. Universitá degli studio di Verona. Recuperado de http://www.univr.it. Buck Institute for Education (BIE). Recuperado de http://www.bie.org.

\section{REFERENCIA BIBLIOGRÁFICA}

López, Z.: El aprendizaje basado en proyectos (ABP) como metodología de trabajo en el aula universitaria de lengua española con alumnado italiano. Aula de Encuentro, ㄲo2 (20). pp. 171 196. DOI: https://dx.doi.org/10.17561/ae.v20i2.9 
Zósimo López Pena es Profesor ayudante en la Facultad de Educación de la Universidad Internacional de La Rioja (UNIR) Correo-e:zosimo.lopez@unir.net 